\title{
A case of mislabeled textbooks: misnomer of the traditional "bicaval" view
}

\author{
Laura Vaskelyte $^{1}$, Stefan Bertog ${ }^{1,2}$, Ilona Hofmann ${ }^{1}$, Bojan Jovanović ${ }^{1}$, Markus Reinartz ${ }^{1}$, Predrag Matic ${ }^{1}$, \\ Sameer Gafoor ${ }^{1,3}$, Kolja Sievert ${ }^{1}$, Horst Sievert ${ }^{1}$ \\ ${ }^{1}$ CardioVascular Center Frankfurt, Frankfurt, Germany; ${ }^{2}$ Minneapolis Veterans Affairs Medical Center, Minneapolis, USA; ${ }^{3}$ Swedish Heart and \\ Vascular Institute, Seattle, USA \\ Correspondence to: Prof. Dr. Horst Sievert. CardioVascular Center Frankfurt, Seckbacher Landstrasse 65, 60389 Frankfurt, Germany. \\ Email: info@CVCFrankfurt.de.
}

\begin{abstract}
Since the early beginning of the transesophageal echocardiography (TEE) era, standardized tomographic views describing cardiac key structures have been provided. They have become the basis of TEE and have not been modified for decades. During our recent structural interventional cases, it has come to our attention that the structure frequently labeled "inferior vena cava" in textbooks and journal articles illustrating the bicaval TEE view is, in fact, the coronary sinus. Our manuscript illustrates our observation.
\end{abstract}

Keywords: Transesophageal echocardiography (TEE); bicaval view; coronary sinus inferior vena cava (CS IVC)

Submitted Feb 01, 2018. Accepted for publication Apr 16, 2018.

doi: $10.21037 /$ cdt.2018.05.01

View this article at: http://dx.doi.org/10.21037/cdt.2018.05.01

Echocardiography is one of the most important noninvasive diagnostic tools in cardiology, providing information on cardiac structures, function and hemodynamics. After Inge Edler and Helmuth Hertz described M-Mode echocardiography in 1953, a new diagnostic era began. The first academic course on cardiac ultrasound and echocardiography textbook and the term "echocardiography" were developed in the 1960s and 1970s (1). Transesophageal echocardiography (TEE) was first described in 1980 (2). The modern era of TEE started after Hanrath et al. attached a phased-array ultrasound transducer to the tip of a flexible gastroscope (3). Today, using multiplane imaging capabilities and real-time $3 \mathrm{D}$ imaging, exact live information about cardiac structures and hemodynamics is obtained and allows diagnosis of complex cardiac anatomy and guidance during interventional and surgical cardiac procedures.

Since the early beginning of the TEE era, standardized tomographic views describing cardiac key structures have been provided. They have become the basis of TEE and have not been modified for decades. In the following, we would like to challenge the popular concept of the traditional "bicaval" view.

\section{What do we see in the "bicaval" view?}

The traditional "bicaval" view is usually obtained from a mid-esophageal position with a transducer angle of $70-110^{\circ}$. This view has been adopted as an integrative part of TEE examinations and intra-procedural imaging. For example, it is widely used for guidance of transseptal punctures. In this view (Figure 1), the left (on the top) and the right (at the bottom) atrium are seen. The interatrial septum (IAS) with the fossa ovalis separates both atria. On the right side of the screen, below the superior aspect of the septum secundum, the superior vena cava (SVC) is located. On the left side of the screen, below the inferior aspect of the septum secundum, is a structure that has traditionally been labeled as the inferior vena cava (IVC) in a number of publications and textbooks leading to the term "bicaval view" (4-6). However, comprehensive multiplane scanning, particularly using catheters and devices from the femoral venous approach allowed us to characterize this structure as the coronary sinus (CS) (Figure 1). The IVC, in fact, usually enters the right atrium at an angle. Figure 2 demonstrates the relationship of the IVC with the right atrium. A wire is placed into the SVC coming from the IVC (via the 


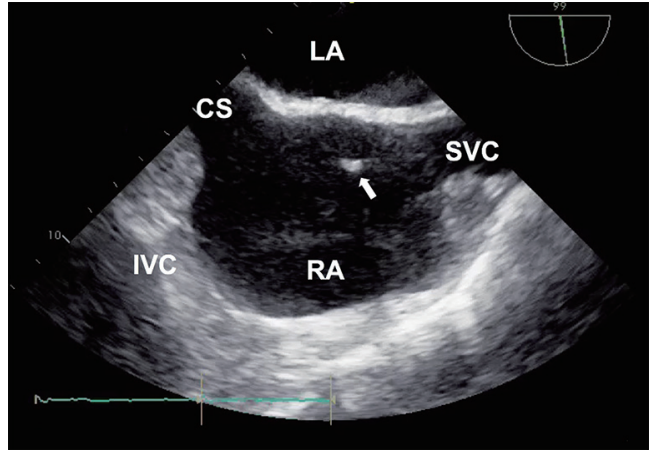

Figure 1 Traditional "bicaval" view at $90-120^{\circ}$ where the CS is frequently labeled as IVC (white arrow: J-tipped wire). LA, left atrium; SVC, superior vena cava; CS, coronary sinus; RA, right atrium.

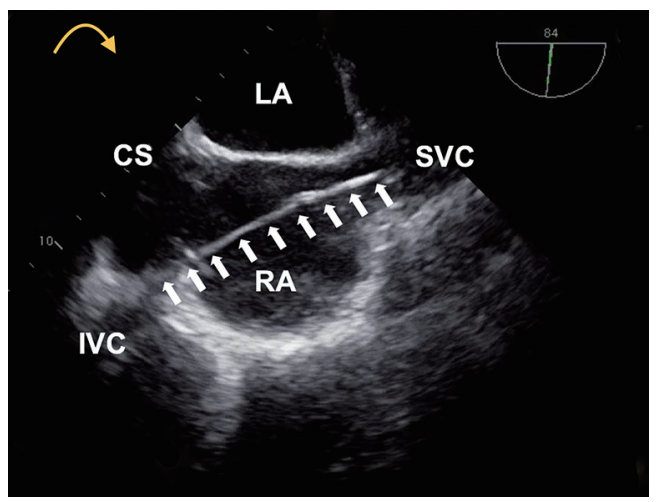

Figure 2 This is the same patient as in Figure 1. In this case the probe was rotated clockwise (yellow arrow) and the transducer angle decreases slightly. It demonstrates that the IVC enters the right atrium at an angle. As proof, a J-tipped wire advances via the femoral vein can be seen entering the right atrium via the IVC and continuing into the SVC. White arrows: J-tipped wire from the femoral vein via the IVC and RA into the SVC. LA, left atrium; IVC, inferior vena cava; SVC, superior vena cava; RA, right atrium.

femoral vein). The IVC enters the right atrium at a nearly $90^{\circ}$ angle. From the typical "bicaval" view, both atria, the IAS, SVC and the CS can be readily identified in one single view (Figure 1). However, it typically requires more probe manipulation [posterior (clockwise) rotation] to visualize

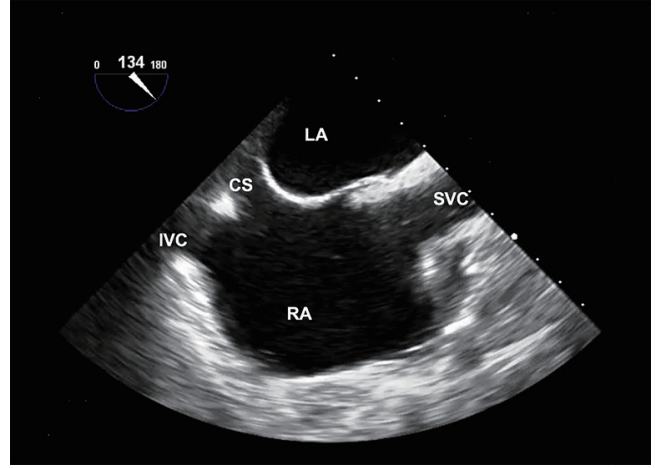

Figure 3 A modified view at $130^{\circ}$ showing both vena cavas and the CS. IVC, inferior vena cava; SVC, superior vena cava; CS, coronary sinus; LA, left atrium; RA, right atrium.

the IVC and it can be tricky to find one plane showing both vena cavas, a true bicaval view (Figure 3). Another view showing the IVC could be found in a modified $0^{\circ}$ plane, from the four-chamber view pulling the probe slightly back and turning posteriorly (clockwise) until the ventricles disappear and the roof of atria emerges. Further supporting our findings are images obtained in a patient with cardiac resynchronization therapy (CRT). The CS lead is shown entering the CS via the right atrium and from the SVC. The CS is seen immediately adjacent to the IAS whereas the IVC enters the right atrium at a slight angle (Figure 4). Similarly, the relationship of the IVC and CS to the right atrium is illustrated in Figure 5.

Recognition of views demonstrating specific cardiac structures is essential allowing quick orientation during structural interventional procedures. Aside from wellestablished procedures on the left side of the heart, there is a growing interest for right-sided procedures such as interventions to the tricuspid valve. An understanding of the right heart anatomy as well as ability to demonstrate key structures is crucial both for pre-procedural planning and for procedural guidance. Once again, the traditional "bicaval" view demonstrates, aside from both atria and IAS, the SVC and CS. Visualization of the IVC, on the other hand, requires modification of the "bicaval" view by turning the TEE probe posteriorly while sometimes slightly reducing the angle of the imaging plane. 

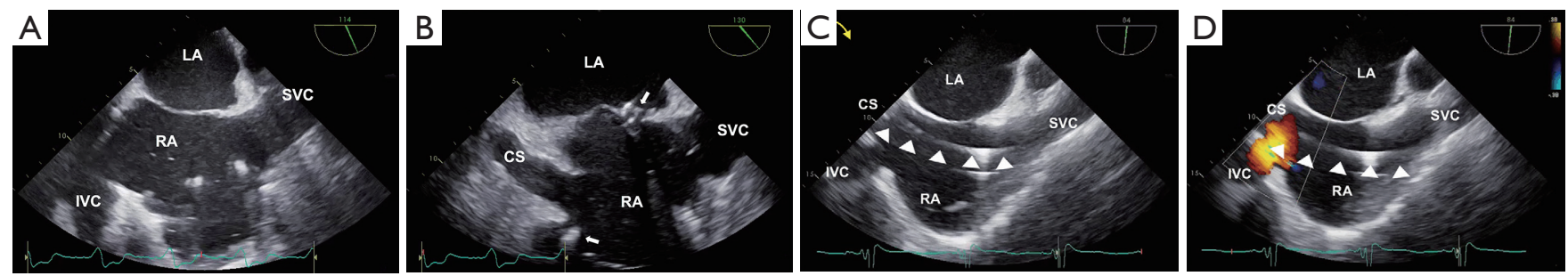

Figure 4 Images obtained in a patient during the transseptal puncture. (A) A view at $110^{\circ}$ showing the both vena cavas; (B) a view showing the SVC and the CS entering into RA with the different angle than the IVC (the tip of the transseptal puncture system is seen at the superior aspect of the fossa ovalis). As proof of aforementioned description, the CS lead is seen entering the CS; (C,D) to visualize it a clockwise rotation of TEE probe (yellow arrow) and slightly reduction of transducer angle was needed (C) and flow demonstrated from the IVC (D). White arrows: transseptal puncture system; white arrow heads: cardiac resynchronization therapy (CRT) lead. IVC, inferior vena cava; SVC, superior vena cava; CS, coronary sinus; LA, left atrium; RA, right atrium.
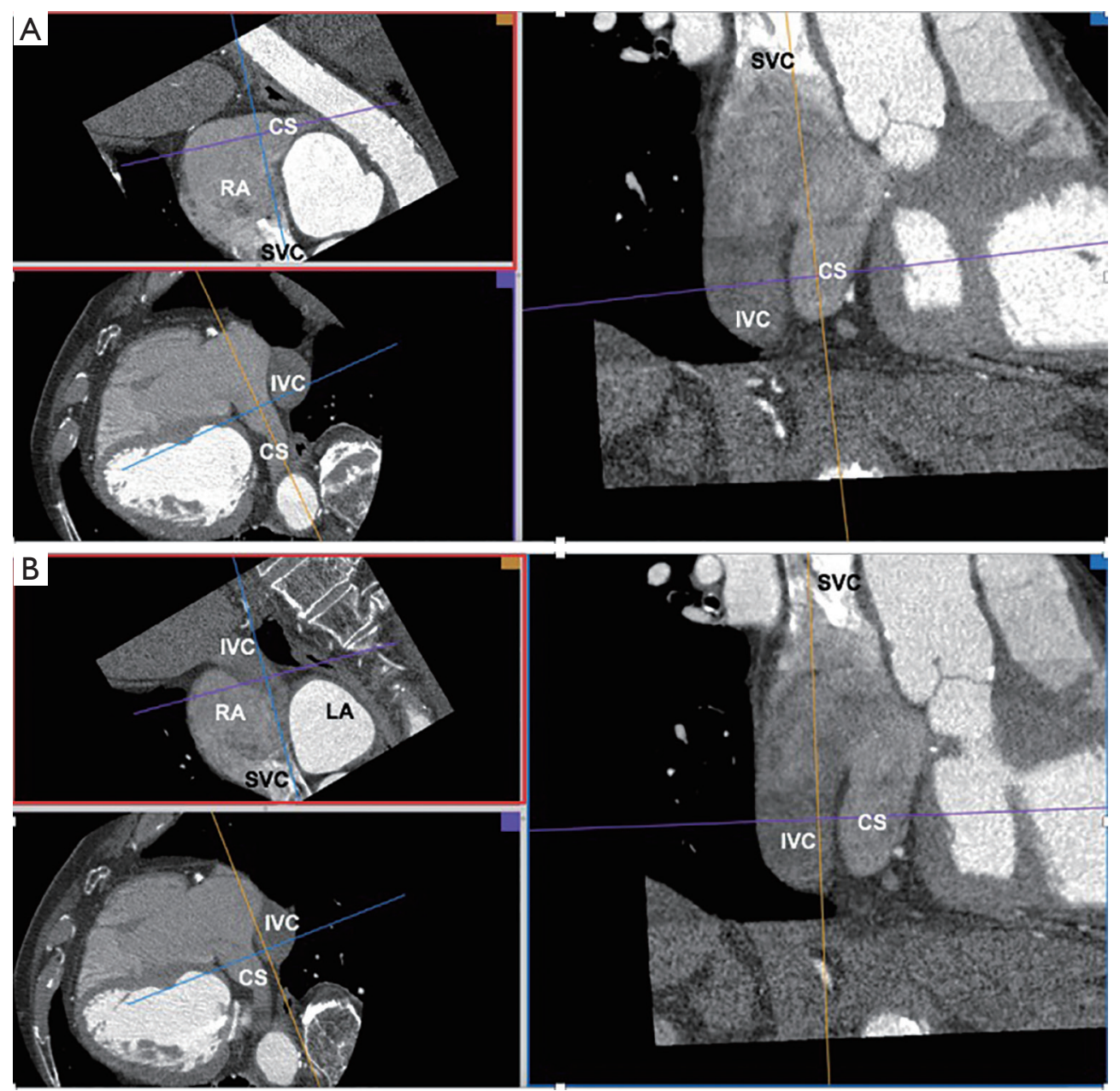

Figure 5 Computed tomography (CT) with multiplane reconstruction (A,B). IVC, inferior vena cava; SVC, superior vena cava; CS, coronary sinus; LA, left atrium; RA, right atrium. 


\section{Acknowledgements}

None.

\section{Footnote}

Conflicts of Interest: The authors have no conflicts of interest to declare.

Informed Consent: Informed consent was obtained from the patients for publication of this manuscript and any accompanying images.

\section{References}

1. Feigenbaum H. Evolution of echocardiography.

Cite this article as: Vaskelyte L, Bertog S, Hofmann I, Jovanović B, Reinartz M, Matić P, Gafoor S, Sievert K, Sievert $\mathrm{H}$. A case of mislabeled textbooks: misnomer of the traditional "bicaval" view. Cardiovasc Diagn Ther 2018;8(4):508-511. doi: $10.21037 /$ cdt.2018.05.01
Circulation 1996;93:1321-7.

2. Hisanaga K, Hisanaga A, Nagata K, et al. Transesophageal cross-sectional echocardiography. Am Heart J 1980;100:605-9.

3. Hanrath P, Kremer P, Langenstein BA, et al. Transesophageal echocardiography. A new method for dynamic ventricle function analysis. Dtsch Med Wochenschr 1981;106:523-5.

4. Raut MS, Maheshwari A. Imaging coronary sinus in modified bicaval view. Ann Card Anaesth 2015;18:430-2.

5. Sidebotham D. Practical perioperative transesophageal echocardiography: with critical care echocardiography. 2nd edition. Philadelphia, PA, USA: Elsevier/Saunders, 2011.

6. Belham M. Transesophageal echocardiography in clinical practice. London, UK: Springer, 2010. 